\title{
GENERATING APPLICATION TO HANDLE SNAPSHOTS IN GOOGLE CLOUD PLATFORM
}

\author{
Aditya Sachin Karmalkar \\ Department of Computer Science and Engineering \\ Walchand College of Engineering \\ Sangli, Maharashtra, India
}

\begin{abstract}
In this paper, we present the system for managing the snapshots on the Google Cloud Platform (GCP) environment using a scheduling mechanism to assist the people to recover their data during data failure or disaster. The usage of cloud services has been increasing and along with that there is a need to protect data in the cloud. The approach of our system adopts usage of the snapshot to manage and protect the data present in disks of the virtual machines in google cloud using scheduling and tag- based scheduling mechanism for snapshots. This will help users during disaster situation to protect and restore their data present in the Google cloud.
\end{abstract}

Keywords- Cloud Computing, Snapshots, Virtual Machine, Disaster recovery.

\section{INTRODUCTION}

The usage of cloud services is increasing due to its offerings of services, better efficiency and performance, cost effective and flexible resources which we can scale up and down as per our needs. As all services are offered remotely it results in reducing cost of physical infrastructure. Cloud has "pay as you use" pricing models, it means we must only pay for resources we have used. Due to its offerings and services most of the organizations are shifting to use clouds.

In Cloud environment, Data storage and management is main factor. Data must be backed-up and stored separately so that it can be used at the time of data loss, data failure issue. Due to server failure many issues can arise also it may lead to failure of business which results in loss. To recover the data or to prevent any loss of data, backup and disaster recovery mechanism is carried out. So generating backup of data frequently will help in business continuity. At this time snapshot comes in picture.

Snapshot is referred as the state or picture of the data present in the volume of instance of virtual machine at a specific time. Snapshots plays crucial role in rolling back the system to a particular time. Snapshots are triggered in a timely manner to record the state of data at that moment and no any data is missed out. During disaster or data loss incident, snapshot can be used to restore the data, the volume will return to the exact state as it was when the snapshot was being taken. Snapshots comes into play when we need to protect the data frequently.

\author{
Dr. Bashirahamad F Momin \\ Department of Computer Science and Engineering \\ Walchand College of Engineering \\ Sangli, Maharashtra, India
}

Snapshots generation can be set for once in an hour or also they can be set within minutes.

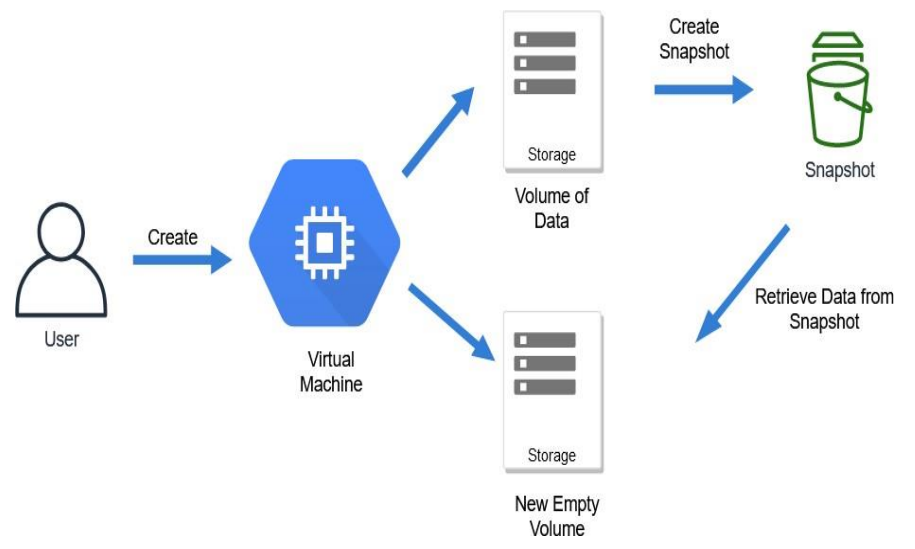

Fig. 1. Snapshot Workflow

Figure 1 gives the idea of working of snapshot and how the data can be restored using snapshots. User needs to create a virtual machine and he may store data in the volume of that particular instance created. Now we will take the snapshot of the data present in the volume. Later if any disaster occurs and to recover from it user will create new instance of virtual machine and in its volume he will restore the data present in the volume of earlier instance by using the snapshot. This is how snapshots helps to gain back the data in case of any disaster or data loss.

The rest of the paper is organized as follows. Proposed methodology is explained in in section III. Experimental results are presented in section $\mathrm{V}$. Concluding remarks are given in section VII.

\section{LITERATURE REVIEW}

Using VNsnap [1] to snapshots of virtual networked infrastructures. It consists of virtual machines connected by virtual network. This paper proposes a model VNsnap which will take snapshot of whole VNI which will also include the images of VM, execution of VM, communication and state of storage. It will be taking the distributed snapshots of VNI which will help in restoring the entire virtual infrastructure. 


\section{International Journal of Engineering Applied Sciences and Technology, 2021 Vol. 6, Issue 2, ISSN No. 2455-2143, Pages 101-104 \\ Published Online June 2021 in IJEAST (http://www.ijeast.com)}

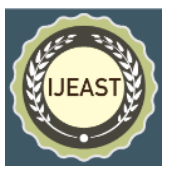

Proposed model VNsnap does not require any modification to application, operating system running in Virtual machine and also it was capable of taking snapshots parallel along with the operations of VNI thus it used to have very less downtime, normally oy few seconds. Distributed snapshot algorithm was adapted by this model to maintain consistency in VM snapshots.

This study [2] focuses on unlimited storage provided by cloud and to analyze the storage for its performance and measure speed while retrieving data. Analysis also helped towards betterment and evaluation for better storage depending on the speed of retrieval of data of different size. They proposed a storage-as-a-service solution in which on different cloud providers experiments were carried out in different time spans using files of varying sizes. It helped to obtain read and write performance of different cloud services and also they aim to evaluate the services which are available because it will lead to better storage methods for clouds.

\section{A. Types of Snapshot Consistency}

- Application consistent: - Application consistent snapshots are considered more reliable but for this snapshot to generate, processing of applications needs to be halt temporarily. It causes interruption in process. Windows VSS (Volume Shadow Copy Services) mechanism is used to generate application consistent copies of snapshots. Before initiating a backup VSS informs every single application to flush the data present in memory to disk. Services are resumed after snapshot is generated.

- File System Consistent: - They take snapshots of all file at the same time to provide the consistency. It records all pending $\mathrm{I} / \mathrm{O}$ operations and data present in memory. Before snapshot is initiated, file system is paused and pending $\mathrm{I} / \mathrm{O}$ operations are flushed to hard drive. This snapshot consistency type cannot match the completeness of application consistent snapshot to record data.

\section{METHODOLOGY}

In our application, at first user needs to register himself by creating his account by providing his google cloud credentials and that must be project id, private key and client email of his cloud account. Initially the credentials will be validated by listing the resources created by user in cloud environment. For validating credentials there is Compute API of GCP which can be used to list resources. If valid credentials are provided then resources are listed and account gets registered in system and user's details will be stored in database and if invalid credentials are provided then error is generated and account is not created. After validation and creation of account, User can even update his account details, credentials and can also delete his account from system.

User can list his resources based on region or labels attached to that resource present in google cloud environment. It will help user to get details of resources. Further by selecting the resource user can trigger snapshot of it. For creation of snapshot, firstly the user may need to create a volume in the VM instance and data must be added in that volume. Later the user must list resources in the system and select the resource and go for snapshot of data present in selected resource at that moment. This will create the snapshot copy of data which can be later use to restore the data present in that copy. User may also go for creating a schedule to generate snapshot of disk. Using this mechanism, Snapshots can be generated after specific time intervals. It will help to keep backup of data present in disk regularly and it will also minimize the risk of data loss or data failure.

If no longer required, user can delete his account from system. Compute resource of GCP has incremental snapshot mechanism because of this every new snapshot will contain the data which has updated or added after the previous snapshot was taken. Newly generated snapshot may require the data stored in older snapshot, they are dependent snapshots. So when a snapshot is deleted doesn't means all data present in snapshot is deleted. Some data is moved to next snapshot and if snapshot is not dependent on any other snapshot then it is deleted directly.

\section{PROBlem STATEMENT}

To Design and Develop a system to manage snapshots of data in Google Cloud Platform.

In today's world Data is considered very important to grow and support your Business, to understand and predict the customer behavior pattern, to plan the upcoming marketing strategies, improve the decision making and to predict the upcoming trends. It is considered as information which can be analyzed to generate conclusion or to derive knowledge from it to make certain effective decisions. Data is of various types and it can be used in various ways by many companies.

Organization may not afford to lose any amount of data. Losing data may put any organization a step behind from market and can also lead to downfall of it. Financial firms and Banking sector will be very much affected if they lose any transaction data.

It is necessary to keep track and backup of data in a periodic manner. So to provide the same we are developing a system which will take snapshots of client's data from the google cloud environment and later at the time of Data Breach, Data Loss or any other Data Failure incident those snapshots can be used to restore the data as it was earlier. The system will have advanced scheduling mechanism to generate the snapshots. Using this mechanism user can set the frequency of 


\section{International Journal of Engineering Applied Sciences and Technology, 2021 \\ Vol. 6, Issue 2, ISSN No. 2455-2143, Pages 101-104 \\ Published Online June 2021 in IJEAST (http://www.ijeast.com)}

hours, days or weeks to generate snapshot automatically. System will also provide tag-based scheduling. It will list all virtual machine instances which has the tag specified by user and then snapshot of all disks attached to those instances can be generated on a single click.

\section{EXPERIMENT AND RESULT}

In this study we are developing an application which is managing and protecting the workload present in google cloud resources by making use of snapshot mechanism. This system is capable to create account by validating user's credentials, store the user's data also it is listing the resources present in google cloud and also this system is generating snapshots of data present in the disks of vm instance. This system allows user to update his details and delete his account if it is not required. Our system will generate error for invalid credentials and also for any operation if it fails to process the request. As a result this application will be efficient for user to manage his data across google cloud and also it will be helpful to manage the snapshots present in it. This system will help to maintain the business continuity.

\section{A. Cloud Account Configuration-}

User will share his google cloud credentials with the application to create account in it. Application will validate the credentials using Compute API and for invalid credentials error will be given and account will not be created. Once the account is created the details of user will be stored in database. Later user can update his details and he can also choose to delete his account from our application.

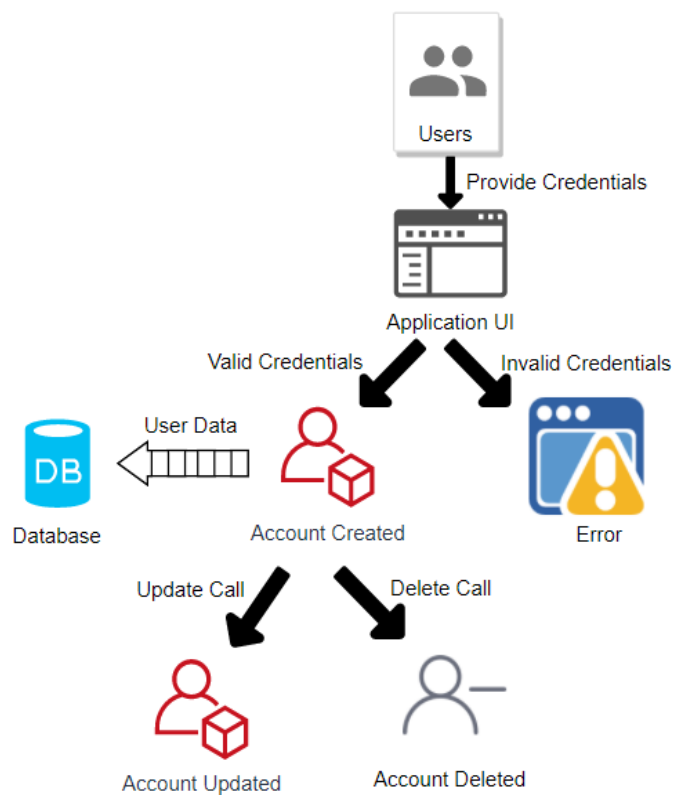

Fig. 2. Account Configuration

\section{B. Listing of VMs -}

Once the account is created in application then user can select his account and list the virtual machines based on the region he has selected present in his google cloud account. By listing the resources he will get all the details of VMs and he can select the VMs to trigger the snapshot.

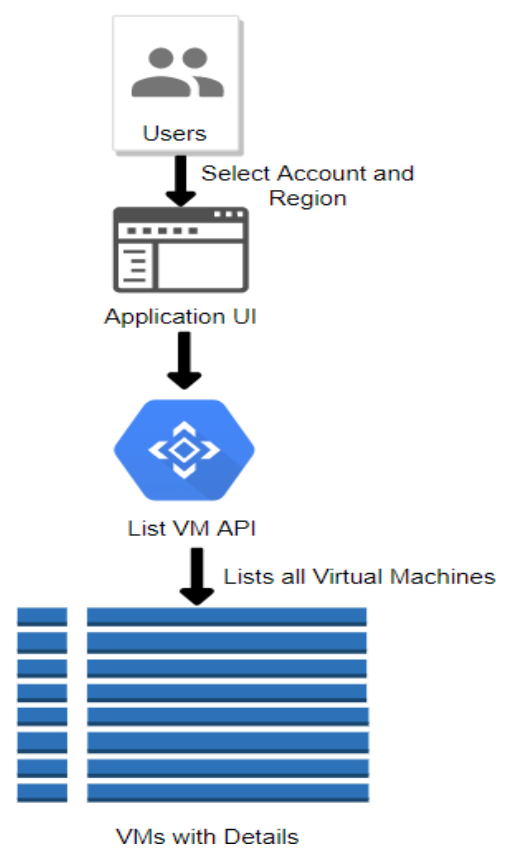

Fig. 3. Resource Listing

\section{Generate Snapshot -}

After listing the VMs user can select one or many VMs at once to generate the snapshot of the data present in the volumes of selected VMs. User can also select VMs from different region to generate snapshot.

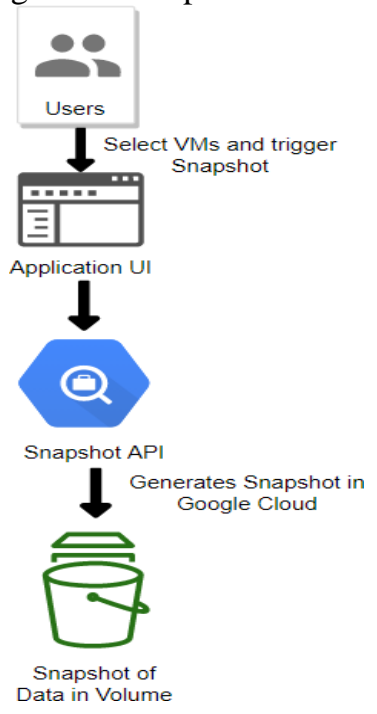

Fig. 4. Snapshot Creation 


\section{International Journal of Engineering Applied Sciences and Technology, 2021 \\ Vol. 6, Issue 2, ISSN No. 2455-2143, Pages 101-104 \\ Published Online June 2021 in IJEAST (http://www.ijeast.com)}

This study discuss about the issue of data failure during disaster and the importance of managing data present in cloud environment. For this issue we proposed an application to manage and protect the data present in google cloud using snapshots.

\section{LIMITATIONS}

This application is only capable of handling snapshots of disks in google cloud. It doesn't protect disks of google cloud against malware or any cyber-attacks. Also, it doesn't protect group of machines which are distributed over network using snapshots.

\section{CONCLUSION}

It is observed that system presented in this study will assist the user in managing the data workload across the GCP resource using the snapshot mechanism. This will help user to manage data present in cloud during any data loss or data failure incident. It will maintain the business continuity.

The study here is concentrated on managing and protecting data present in compute resource of GCP. Looking into the current study, this information can be useful for reference of any future study.

\section{REFERENCE}

[1] Ardalan K., Patrick E., Dongyan X., "VNsnap: Taking Snapshots of Virtual Networked Infrastructures in the Cloud“, In IEEE Transactions on Services Computing ( Volume: 5, Issue: 4, Fourth Quarter 2012), (pp. 484-496)

[2] V. Abhishek and S. N. Megha, "Cloud Storage and Retrieval - A User Perspective," In 2014 World Congress on Computing and Communication Technologies, 27 Feb.-1 March 2014, 978-1-4799-2877-4.

[3] Stephanie C., Faiez Z., Christophe G., Philippe M., “A Precise Model for Google Cloud Platform” In 2018 IEEE International Conference on Cloud Engineering (IC2E), 17-20 April 2018 0-7695-6371-6/18.

[4] Getting Started with Google Cloud. https://cloud.google.com/gcp/getting-started

[5] Google Cloud Platform - Documentation. https://cloud.google.com/docs

[6] How Snapshot works? https://searchdatabackup.techtarget.com/feature/Everythin g-you-need- to-know-about-snapshotting

[7] Details of different credentials in GCP https://cran.r-

project.org/web/packages/gargle/vignettes/get-apicredentials.html

[8] Application-Consistent https://www.unitrends.com/blog/application-consistencyagent-agentless-protection-virtual-machine-backups

[9] File-System Consistent https://www.synology.com/englobal/knowledgebase/DSM/tutorial/Virtualization/What_ is_file_system_consistent_snapshot. 